C A S E R E P O R T

\title{
An inverted uterine fundus carrying placenta: How does this happen or why is it mistaken?
}

\author{
Rashmi Chaudhary, Geeta Gurung, Archana Amatya, \\ Kamala Saki, Shreejana Maharjan. \\ Dept of Obs/Gyn, TUTH, Kathmandu, Nepal
}

\begin{abstract}
A second gravida with normal antenatal period delivered a baby within an hour of admission and also expelled placenta which appeared at vagina. This placenta was adhererent to the fundus of uterus which was inverted but was persistently pulled out as uterine inversion was unrecognized. This case report will be of help to many of us who may mistake such condition. A habit to palpate the fundus of uterus and make sure it is contracted before controlled cord traction will help making the diagnosis in time.
\end{abstract}

Keywords. Controlled cord traction, fundal attachment of placenta, uterine inversion

\section{Introduction}

It is rare to see a case of uterine inversion or find the inverted fundus carrying the placenta at the time of inversion, which has been opined to have a protective effect against the development of shock. ${ }^{1,2}$ A case reported below describes how an inverted uterus carrying morbidly adherent placenta was complicated by hemorrhagic shock when mistaken for normal placental delivery.

\section{Case}

A 29 years old G2 P1 with a history of normal vaginal delivery 3 years back was booked at our antenatal clinic. She had normal antenatal period and was admitted in active stage of labor at 38 weeks of pregnancy. After 40 minutes of arrival, she delivered a baby girl spontaneously, in a pool of thick meconium stained liquor. Unfortunately, all other team simultaneously occupied in emergency, this being one of the busiest night duties with six caesarean sections in row, the staff who conducted the delivery had to rush to resuscitate the baby.

When she came back to the parturient mother she noticed that the placenta was occupied around the

Correspondence

Geeta Gurung, DGO, FCPS

Assoc. Prof. Dept. of Obs/Gyn, TUTH, Kathmandu, Nepal

Email:gurunggeeta@hotmail.com vaginal orifice (Fig 1). She also realized that the normal time limit of two minutes set for administration of oxytocin outlined in the active management of second stage of labor had already exceeded. Although the placenta appeared in vagina, it could not be delivered so she asked for help from Resident doctor who was engaged in ringing Paediatrician to make consultation for the new born baby for meconium aspiration. She was engrossed in placental delivery and after futile attempts; senior doctor was called for who also got indulged in removing partially separated placenta, before recognizing the condition to be uterine inversion. After making the diagnosis of acute uterine inversion, patient was immediately shifted to Operation Theatre. Under General Anesthesia morbidly adherent placenta attached to the endometrium of the inverted fundus of uterus was removed before manually reverting or repositioning the uterus. Methergin I/V, oxytocin I/V infusion and prostaglandin $\mathrm{F}_{2}$ a intra myometrial were given to control hemorrhagic shock $(>1000 \mathrm{ml}$ of blood loss) further necessitating ICU management for ventillatory support. Dopamine, adrenaline and 7 units of blood and blood products [whole blood transfusion (2); platelet rich plasma (3) and fresh frozen plasma (2)] brought an obvious improvement after 48 hours when she was shifted to maternity ward. Raise was observed in hemoglobin $(7.2-10.1 \mathrm{gm} \%)$ and platelets count $(31,000 / \mathrm{cmm}$ to $121,000 / \mathrm{cmm})$. 


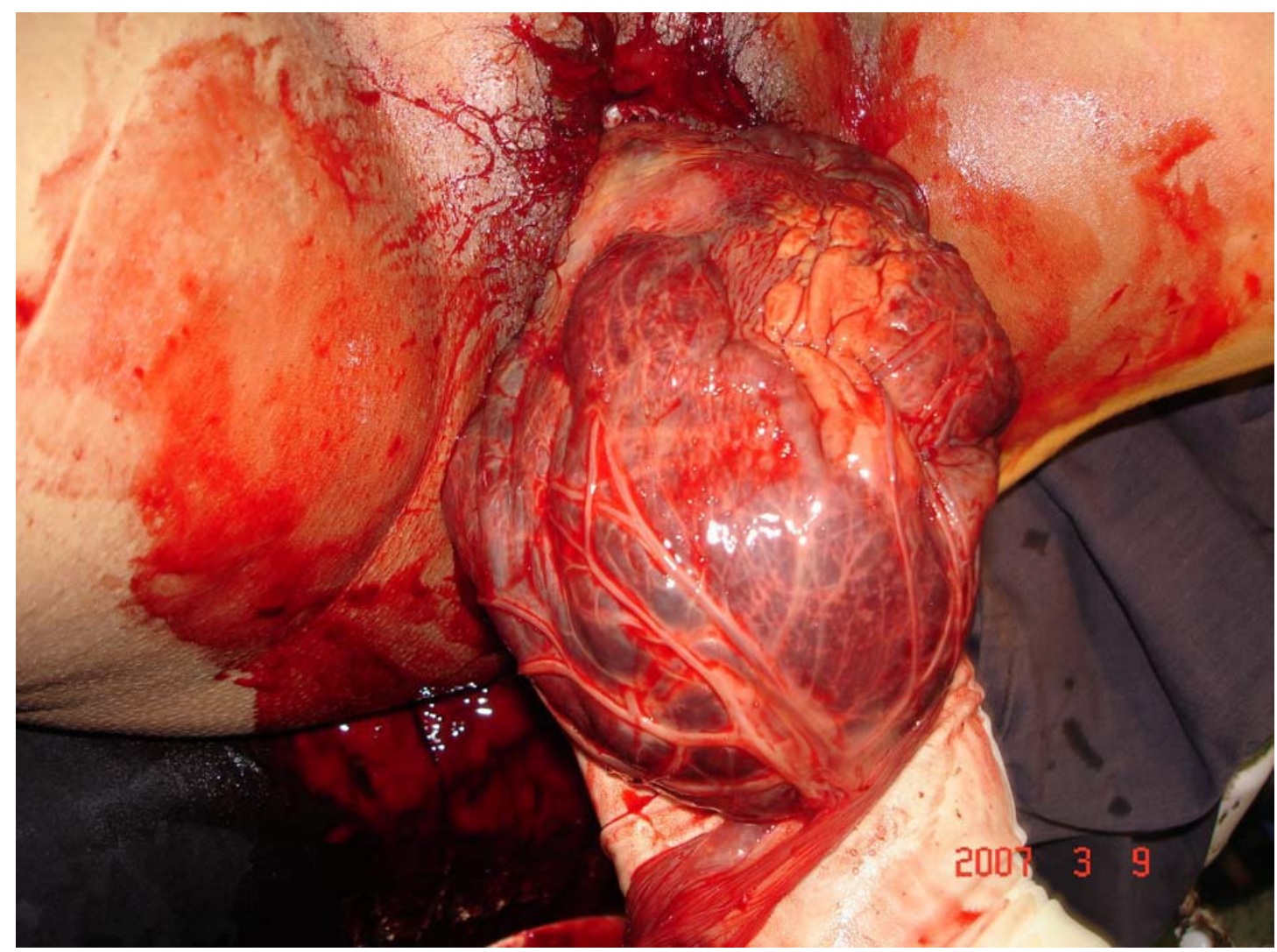

Figure I. Morbidly adherent plocenta on to the fundus

On 7th day, Psychiatric consultation was made for postpartum depression and was managed with counseling daily. On $12^{\text {th }}$ day manual vacuum evacuation was done obtaining $50 \mathrm{~g}$ of placental tissues detected by ultrasound. She was discharged the next day.

\section{Comment}

It is natural not to recognize uterine inversion as this condition has become very rare after the active management of labor. ${ }^{2-4}$ The principle problem in this case was delay in the administration of oxytocin and the coincidence of fundal insertion of placenta which became an additive factor in promoting inversion over a relaxed uterus. Other contributing for uterine inversion was mothers own expulsive effort as a matter of unassisted third stage.

Missing the diagnosis of uterine inversion was the next unpardonable mistake which could have been simply avoided by detecting the absence of uterine fundus abdominally. Instead, the placental delivery was approached, without elevating the uterus, which is the most essential part in the management of third stage of labour.
This indicates that all the Residents and Staff nurse, before they take up labor room posting need to be educated about uterine inversion which is a life threatening condition.

Regarding the management, let us be clear in one point. Should the placenta be manually removed before or after reverting the uterus? Inadvisability of removing the placenta prior to replacement has been demonstrated. ${ }^{2}$ This probably holds true, citing our case as an example, where persuasion to deliver placenta without reposing the uterus was complicated by hemorrhagic shock. Furthermore, unforeseen placental adhesion or placenta acrreta could require more time than anticipated thus allowing the formation of inversion ring necessitating laparotomy and surgical intervention..$^{3,4}$

The difficulty of repositioning an inverted uterus with a huge placenta in situ is equally concerning which complicated this case. However one must always try to repose the uterus first in order to avoid the probability of the formation of resilient inversion ring at unnecessarily fiddling. Secondly, by any chance manual removal of placenta is safer in reverted uterus, 
because one does not know whether there is morbid placental adhesion or not and whether hysterectomy could become the mandate. ${ }^{3}$

Though we have successfully managed this case of uterine inversion, it is sad that we could not prevent it. This might have occurred because of atonicity of uterus as oxytocin could not be administered within one to minute of child birth whereas the presence of other factors like fundal attachment of placenta was inherent. Patients own expuslsive effort during the period of uterine atonicity was an example of unsupported third stage of labour management.

Usually person taking the delivery veil misconduct of third stage of labor which is primarily the reason for uterine inversion. ${ }^{5}$ We are honest about it and feel that oxytocin within one minute of delivery of the baby (after ruling out twins) could recover uterine tonicity to make CCT more feasible with the return of uterine contraction [by exerting the slight controlled traction on the clamped umbilical cord with one hand simultaneously elevating the uterus abdominally towards the umbilicus, laying stretched index finger and thumb of the other hand suprapubically, on the opposite direction].

In conclusion one must palpate and feel for full fledged fundus of the uterus near the umbilicus abdominally, since the loss of uterine fundus clearly denotes uterine inversion, a simple way not to misdiagnose uterine inversion, when huge placenta covers the entire endometrial surface of the inverted uterus.

\section{Acknowledgement}

It would be rude not to be apologetic for the unintended complications that arose during the delivery of this case, which could have been avoided. One and everyone one connected with the successful emergent management of this case owe applause, without whose help the maternal survival would have been next to impossible.

\section{References}

1. Lago JD. Presentation of acute uterine inversion in the emergency department. Am J Emerg Med. 1991 May; 9(3):239-42.

2. Brar HS, Greenspoon JS, Platt LD, Paul RH. Acute puerperal uterine inversion. New approaches to management. J Reprod Med. 1989 Feb; 34(2): 173-7.

3. Achanna S, Mohamed Z, Krishnan M. Puerperal uterine inversion: a report of four cases. J Obstet Gynaecol Res. 2006 Jun; 32(3):341-5.

4. Rana A, Pradhan N, Giri K. Bista D, Thakur JK. Acute uterine inversion reverted by laparotomy and division of ring anteriorly, Dobbins operation. Singapore J of Obs/Gyn 2002; 33(3):50-54.

5. Cumming DC, Taylor PJ. Puerperal uterine inversion: report of nine cases. Can Med Assoc J. 1978 May 20; 118(10):1268-70. 